Check for updates

Cite this: Chem. Sci., 2020, 11, 281

๑ All publication charges for this article have been paid for by the Royal Society of Chemistry

Received 31st July 2019

Accepted 8th November 2019

DOI: $10.1039 / c 9 s c 03798 \mathrm{e}$

rsc.li/chemical-science

\section{Physicochemical-property guided design of a highly sensitive probe to image nitrosative stress in the pathology of stroke $\uparrow$}

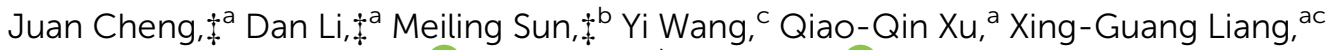 \\ Yun-Bi Lu, ${ }^{c}$ Yongzhou Hu, (D) a Feng Han ${ }^{\star b}$ and Xin Li (D) *a
}

\begin{abstract}
In vivo real-time imaging of nitrosative stress in the pathology of stroke has long been a formidable challenge due to both the presence of the blood-brain barrier (BBB) and the elusive nature of reactive nitrogen species, while this task is also informative to gain a molecular level understanding of neurovascular injury caused by nitrosative stress during the stroke episode. Herein, using a physicochemical property-guided probe design strategy in combination with the reaction-based probe design rationale, we have developed an ultrasensitive probe for imaging nitrosative stress evolved in the pathology of stroke. This probe demonstrates an almost zero background fluorescence signal but a maximum 1000-fold fluorescence enhancement in response to peroxynitrite, the nitrosative stress marker. Due to its good physicochemical properties, the probe readily penetrates the BBB after intravenous administration, and quickly accumulates in mice brain to sense local vascular injuries. After accomplishing its imaging mission, the probe is easily metabolized and therefore won't cause safety concerns. These desirable features make the probe competent for the straightforward visualization of nitrosative stress progression in stroke pathology.
\end{abstract}

Stroke is an acute episode of focal dysfunction of the brain, spinal cord or retina lasting longer than 24 h. $^{1}$ It is one of the most dangerous diseases with a high rate of mortality and disability. Despite recent remarkable advances in its treatment and prevention, stroke remains the second single most frequent cause of death for people older than 60 years and the first cause of permanent disability. ${ }^{2}$ With the aging of the world's population, the global burden of stroke keeps increasing. In consequence, there is a pressing need to continue investigating the mechanism, prevention and treatment of this devastating disease. Several key players contribute to neuronal injury and death following stroke, including oxidative and nitrosative stress, excitotoxicity, and inflammation, ${ }^{3}$ among which, the damaging role of nitrosative stress is attracting increasing research interest. Nitrosative stress is represented by the overproduction of peroxynitrite $\left(\mathrm{ONOO}^{-}\right)$during both cerebral ischemia and subsequent reperfusion., ${ }^{4,5}$ It facilitates the demise of the penumbra by protein nitration, lipid

${ }^{a}$ College of Pharmaceutical Sciences, Zhejiang University, Hangzhou 310058, China. E-mail: lixin81@zju.edu.cn

${ }^{b}$ School of Pharmacy, Nanjing Medical University, Nanjing 211166, China. E-mail: fenghan169@njmu.edu.cn

'School of Medicine, Zhejiang University, Hangzhou 310058, China

$\dagger$ Electronic supplementary information (ESI) available. See DOI: 10.1039/c9sc03798e

$\$$ These authors contributed equally to this work. peroxidation, DNA oxidation, mitochondrial damage, activation or inhibition of various signaling pathways, and blood-brain barrier (BBB) breakdown. ${ }^{6}$ Due to its ultrahigh toxicity, it is emerging as an attractive molecular target for therapeutic intervention. ${ }^{6}$

One of the main challenges in the development of $\mathrm{ONOO}^{-}$targeted stroke treatment is the ideal timing and dosage of medication, as this determines a lot of clinical outcomes. ${ }^{7} \mathrm{~A}$ technology capable of spatiotemporally mapping nitrosative stress during stroke onset and progression would improve people's understanding of when and how $\mathrm{ONOO}^{-}$overproduction contributes to stroke pathology, yielding straightforward information for treatment design. However, this has long been a forbidden task due to the extremely short half-life of $\mathrm{ONOO}^{-}$and its non-genetic origin. ${ }^{8,9}$ Recent advances in reaction-based small-molecule fluorescent probes provide the possibility to track elusive small biomolecules in their local environments. ${ }^{10,11}$ Actually, for targeting $\mathrm{ONOO}^{-}$, there have been several robust probes reported that were confirmed for their specificity and capability to image nitrosative stress in the context of inflammation, ${ }^{12-16}$ kidney injury, ${ }^{17}$ hepatotoxicity ${ }^{18-21}$ and heart disease ${ }^{22,23}$ However, the context of the neurovascular unit is more formidable due to the presence of the blood-brain barrier (BBB). Actually, due to the rigid planar structure of most fluorophores which is essential for their emissive properties, few probes have been reported to readily penetrate the BBB and 
realize the non-invasive imaging of stroke events. Herein, we reported our physicochemical-property guided design of an ultrasensitive fluorogenic probe, which demonstrates desirable brain distribution after intravenous injection to fulfill the direct observation of nitrosative stress during stroke onset and progression.

\section{Results and discussion}

\section{Physicochemical-property guided probe design}

To image local nitrosative stress during stroke onset, it is necessary for the probe to readily penetrate the BBB and accumulate there to a desirable concentration. Similar to the principles in drug development, we assume that the physicochemical properties of a probe should play a key role in determining its in vivo absorption and distribution properties. To rationally design a probe with promising brain absorption and distribution properties, we screened a set of classical fluorescent dyes with sufficient brightness for their partition coefficient between water and oil $(\log P)$, which is the fundamental principle for BBB permeability (Fig. 1a). ${ }^{24} \log P$ measurements were conducted with a shake flask $n$-octanol/water partition experiment. The results showed that among the eight fluorophores commonly used for bio-imaging (Fig. S1 $\dagger$ ), the benzoBODIPY scaffold (F4) showed a $\log P$ of 2.60 which falls into the suggested threshold of 2-5 proposed by Hitchcock et al. for

a. Log $P$ based-screening for fluorophores with potential BBB permeability

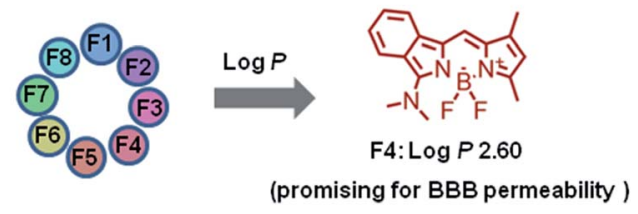

b. Electron-rich phenol as a reaction trigger for ONOO

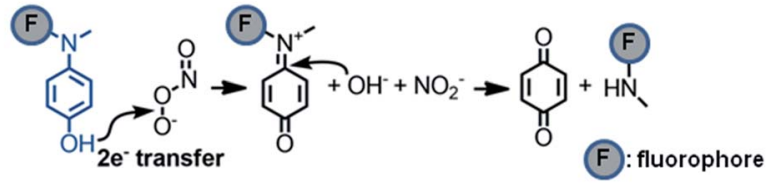

c. Physicochemical-property guided probe design

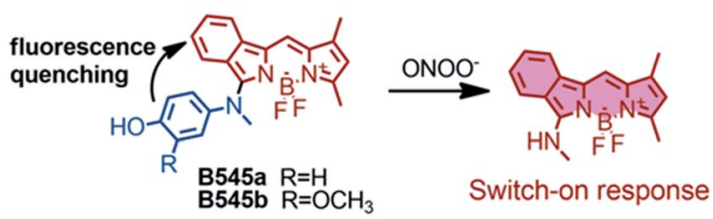

Fig. 1 Physicochemical-property guided fluorescent probe design for non-invasive imaging of $\mathrm{ONOO}^{-}$during stroke in live mice. (a) Partition coefficients $(\log P$ ) of classical fluorophores were determined and benzo-BODIPY (F4) was predicted to have a potentially good BBB permeability. (b) Electron-rich phenols may be oxidized to quinine by $\mathrm{ONOO}^{-}$and this reaction has been used as a trigger for $\mathrm{ONOO}^{-}$probe development. (c) Probes B545a and B545b were designed based on the F4 fluorophore which showed desirable $\log P$ for BBB permeability. B545a and B545b were hypothesized to undergo a biocompatible reaction with $\mathrm{ONOO}^{-}$to be transformed into highly fluorescent products. possible BBB permeability (Fig. 1a). ${ }^{24}$ Moreover, F4 also demonstrated a moderate water solubility of $250 \mu \mathrm{M}$ as determined by the UV-Visible spectrophotometric method (Fig. S2 $\dagger$ ). Besides, F4 was quite bright with a quantum yield $(\Phi)$ of 0.788 in aqueous solution. All these physicochemical and photophysical properties make the F4 fluorophore a desirable starting point for probe development.

Given the reactivity of $\mathrm{ONOO}^{-}$to oxidize electron-rich phenols into quinines which has been utilized with a high degree of success for $\mathrm{ONOO}^{-}$probe design (Fig. 1b), ${ }^{25-30}$ the $p$ hydroxyl aniline moiety was selected as a reaction trigger for $\mathrm{ONOO}^{-}$sensing, and probes B545a and B545b were designed (Fig. 1c). We envisioned that probes B545a and B545b should have little fluorescence due to the free rotation between the fluorophore and the $p$-hydroxyl aniline moiety, while the $p$ hydroxyl aniline moiety should be readily oxidized by $\mathrm{ONOO}^{-}$ under biological conditions, yielding a fluorophore structurally similar to $\mathbf{F 4}$ with strong fluorescence. And this change in probe fluorescence intensity may be recorded as a signal output for $\mathrm{ONOO}^{-}$sensing.

Probes B545a and B545b were facilely synthesized by the nucleophilic substitution of $\alpha$-chloro benzo-BODIPY with the corresponding anilines referring to literature procedures. ${ }^{31}$ Their structures were characterized by both NMR spectroscopy and HRMS analysis.

\section{Validating the reactivity of probes B545a and B545b towards ONOO}

Initially, the performance of the two probes in response to $\mathrm{ONOO}^{-}$was evaluated in PBS buffer $(10 \mathrm{mM}, \mathrm{pH} 7.4)$. As expected, the probes alone were barely fluorescent $(5 \mu \mathrm{M})$, presumably due to the energy consumption caused by the free rotation of the phenol ring around the BODIPY ring (Fig. S3a $\dagger$ ). However, the addition of $\mathrm{ONOO}^{-}(10 \mu \mathrm{M})$ triggered almost a simultaneous fluorescence intensity increase of the two probes, with probe $\mathbf{B 5 4 5 b}$ giving a more dramatic increase (741fold) than B545a (5-fold) (Fig. S3b $\dagger$ ). We presume that the better sensitivity of $\mathbf{B 5 4 5 \mathbf { b }}$ is due to the additional methoxyl group ortho to the hydroxyl group which is electron-donating and in consequence makes the electron transferring reaction towards $\mathrm{ONOO}^{-}$more efficient. B545b was then selected as a candidate probe for $\mathrm{ONOO}^{-}$detection and its responsive features were studied in detail under biologically relevant conditions (PBS, $10 \mathrm{mM}, \mathrm{pH}$ 7.4).

First, changes in the absorptive spectra of B545b in response to various doses of $\mathrm{ONOO}^{-}$were examined. As shown in Fig. 2a, B545b showed its maximum absorption at $508 \mathrm{~nm}(\varepsilon 23350)$ attributed to the $\pi \rightarrow \pi^{*}$ absorption of the BODIPY core. However, the intensity of this band decreased gradually with increasing dose of $\mathrm{ONOO}^{-}$. And a slight blue shift of the maximum absorbance wavelength (498 nm) was observed, suggesting that the benzo-BODIPY scaffold remained largely intact under this condition while the newly formed substitution demonstrated a lower auxochromic effect compared to the $p$ hydroxyl aniline moiety. This observation agrees with the proposed dearylation reaction of $\mathbf{B 5 4 5 b}$ induced by $\mathrm{ONOO}^{-}$. 

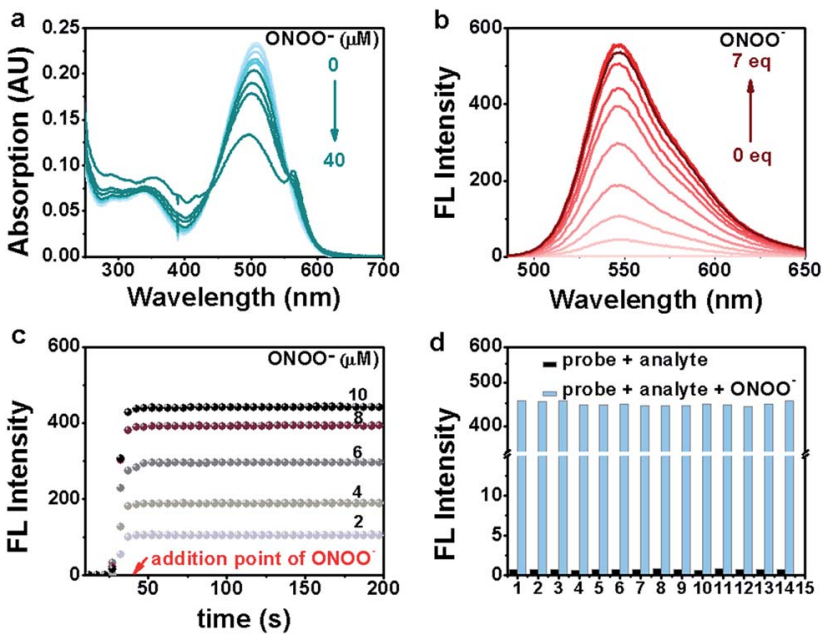

Fig. 2 UV-Vis and fluorescence response of probe $\mathrm{B} 545 \mathrm{~b}$ to $\mathrm{ONOO}^{-}$ (a) Absorption spectra of B545b $(10.0 \mu \mathrm{M})$ before and after being treated with various amounts of $\mathrm{ONOO}^{-}$. (b) Fluorescence spectra of probe $\mathrm{B} 545 \mathrm{~b}(5.0 \mu \mathrm{M})$ before and after treatment with $\mathrm{ONOO}^{-}$at indicated final concentrations. (c) Time-lapse emission of B545b $(5.0$ $\mu \mathrm{M})$ at $545 \mathrm{~nm}$ in response to $\mathrm{ONOO}^{-}$. (d) Fluorescence intensity of B545b $(5.0 \mu \mathrm{M})$ at $545 \mathrm{~nm}$ after treatment with various biologically related species. (1) Probe blank, (2) NO, (3) $\mathrm{NO}_{2}{ }^{-}$, (4) $\mathrm{NO}_{3}{ }^{-}$(5) $\mathrm{H}_{2} \mathrm{O}_{2}$, (6) THBP, (7) ${ }^{\circ} \mathrm{OH},(8){ }^{1} \mathrm{O}_{2}$ (9) $\mathrm{O}_{2}{ }^{-}$, (10) $\mathrm{ClO}^{-}$, (11) $\mathrm{GSH}$, (12) Cys, (13) $\mathrm{Zn}^{2+}$, and (14) $\mathrm{Cu}^{2+}$. All analytes were kept at a final concentration of $100 \mu \mathrm{M}$ except $\mathrm{ONOO}^{-}$which was kept at $10 \mu \mathrm{M}$. All measurements were carried out at ambient temperature with excitation at $475 \mathrm{~nm}$ and emission at $545 \mathrm{~nm}$

Then, the fluorescence titration experiment was performed, and an increase of B545b emission intensity was observed after $\mathrm{ONOO}^{-}$treatment. The increase was $\mathrm{ONOO}^{-}$dose-dependent (Fig. 2b). Plotting the B545b emission intensity at $545 \mathrm{~nm}$ versus $\mathrm{ONOO}^{-}$concentration gave an exponential dependence (Fig. S4†), suggesting a positive correlation between the $\mathrm{ONOO}^{-}$ concentration and $\mathbf{B 5 4 5 b}$ fluorescence intensity. Notably, even a trace amount of $\mathrm{ONOO}^{-}$as low as $50 \mathrm{nM}$ could still trigger an 8 -fold enhancement of B545b emission intensity (Fig. S5 †). An $\mathrm{ONOO}^{-}$concentration as low as $0.5 \mathrm{nM}$ can induce a statistically significant increase of B545b fluorescence (Fig. S6†). All these results suggest the high sensitivity of B545b, supported by both its weak background signal and the high quantum yield of the $\mathbf{F} 4$ fluorophore.

Next, the kinetic properties of the detection reaction were studied. For this purpose, B545b fluorescence was recorded in a time-lapsed way before and after $\mathrm{ONOO}^{-}$treatment. An immediate increase of B545b fluorescence was observed as $\mathrm{ONOO}^{-}$was added, and the increase plateaued in 10 seconds (Fig. 2c), suggesting the ultrafast detection kinetics of B545b for $\mathrm{ONOO}^{-}$.

The selectivity of $\mathbf{B 5 4 5 b}$ for $\mathrm{ONOO}^{-}$was tested by recording its fluorescence response towards various biologically related species especially reactive oxidative species. It turned out that only $\mathrm{ONOO}^{-}$could switch on the fluorescence of $\mathbf{B 5 4 5 b}$ (Fig. 2d). It is noteworthy that B545b could still respond to $\mathrm{ONOO}^{-}$with a similar degree of fluorescence enhancement even in the presence of other biologically related species, suggesting its potential applicability for detecting $\mathrm{ONOO}^{-}$in a complex environment.

As the $\mathrm{H}^{+}$concentration is modestly increased in ischemic tissues in early stages of ischemia as a result of lactic acidosis which leads to a decrease in $\mathrm{pH}$ to $6.4-6.7,{ }^{32}$ it is therefore necessary to check the effect of surrounding $\mathrm{pH}$ on the detection sensitivity of B545b. As shown in Fig. S7, $\dagger$ B545b in various $\mathrm{pH}$ surroundings responded to a set dose of $\mathrm{ONOO}^{-}$with a similar degree of fluorescence increase, suggesting that $\mathbf{B 5 4 5 b}$ should be able to image $\mathrm{ONOO}^{-}$with a similar degree of sensitivity in the acidified bio-microenvironments.

Finally, after confirming the sensitive and selective fluorescence switch-on response of $\mathbf{B 5 4 5 b}$ to $\mathrm{ONOO}^{-}$, we monitored the detection reaction with a liquid chromatograph equipped with a mass detector (LC-MS) to make further confirmation on the detection mechanism. Treating $\mathbf{B 5 4 5 b}$ with $\mathrm{ONOO}^{-}$caused an $\mathrm{ONOO}^{-}$-dose dependent decrease of the $\mathbf{B 5 4 5 b}$ signal in the chromatogram while the emergence of a peak whose molecular weight agrees well with the proposed detection product in Fig. 1c (Fig. S8-S10†).

\section{B545b safety and BBB permeability evaluation}

Having confirmed the good performance of probe B545b as a fluorogenic probe for sensing $\mathrm{ONOO}^{-}$in aqueous solution, we then tested its cytotoxicity and BBB permeability as a further evaluation of its bio-imaging applicability. With a CCK8 assay, B545b was found to cause a negligible effect on the viability of EA.hy926 cells (Fig. S11†), implying its safety for bio-imaging.

The $\log P$ of B545b was measured to be 2.7 by the shake flask $n$-octanol/water partition experiment, which, as expected, falls into the scope of 2-5 proposed by Hitchcock et al. for possible BBB permeability. ${ }^{24}$ To make further confirmation, the BBB permeability of $\mathbf{B 5 4 5 b}$ was determined with the classical pharmacokinetic techniques (intravenous administration and tissue sampling)..$^{33}$ Mice were administered with B545b $\left(5 \mathrm{mg} \mathrm{kg}^{-1}\right)$ via intravenous injection, and then sacrificed at different time intervals to measure B545b concentrations in the brain and plasma. As shown in Fig. S12, $\uparrow$ due to its desirable physicochemical properties, B545b accumulates easily in the brain tissue, and a brain-to-plasma concentration ratio of 5 was observed in mice $30 \mathrm{~min}$ after probe administration. Moreover, B545b is easily metabolized. Its plasma and brain concentration dropped significantly in $2 \mathrm{~h}$ after administration. This good brain uptake, but non-retention pharmacokinetic properties, of $\mathbf{B 5 4 5 b}$ is especially desirable, as this should decrease its risk on brain function.

\section{B545b-aided observation of $\mathrm{ONOO}^{-}$formation in endothelial cells}

To test the sensitivity of $\mathbf{B 5 4 5} \mathbf{b}$ to image $\mathrm{ONOO}^{-}$in live cells, we first optimized its working concentration for live cell staining. EA.hy926 cells were first treated with 3-morpholinosydnonimine (SIN-1, $0.06 \mathrm{mM}$ ), an $\mathrm{ONOO}^{-}$donor, ${ }^{34}$ to up-regulate intracellular $\mathrm{ONOO}^{-}$levels. After being stained with B545b of various concentrations for $30 \mathrm{~min}$, cells were observed under a confocal microscope. The results showed that the intracellular B545b fluorescence increased in a probe dose-dependent way, 
and significant brightness was observed at a concentration of $0.5 \mu \mathrm{M}$ (Fig. S13†). Furthermore, B545b at $0.5 \mu \mathrm{M}$ also worked well to image endogenous $\mathrm{ONOO}^{-}$in cells induced by oxygenglucose deprivation stimulation (OGD, 4 hours). ${ }^{35}$ This suggests that a B545b concentration of $0.5 \mu \mathrm{M}$ should be sufficient for imaging experiments (Fig. S14 $\dagger$ ).

Then, the capability of B545b to image various degrees of nitrosative stress in EA.hy926 cells was studied. Cells were first stimulated with various doses of SIN-1 for 60 min to induce various degrees of nitrosative stress, and then were stained with B545b for $30 \mathrm{~min}$. The intracellular B545b fluorescence was observed to increase in a SIN-1 dose-dependent way (Fig. 3a and b), indicating the positive correlation between B545b fluorescence and the degree of nitrosative stress. It is noteworthy that B545b was able to track the dynamic progression of intracellular nitrosative stress induced by SIN-1 stimulation. As shown in Fig. 3c and 3d, when EA.hy926 cells were first loaded with B545b (30 min incubation) and then stimulated with SIN-1, the intracellular B545b fluorescence gradually intensified as SIN-1 stimulation proceeded. These results taken together suggest the sensitivity of $\mathbf{B 5 4 5 \mathbf { b }}$ to image intracellular nitrosative stress.

Next, the selectivity of B545b for $\mathrm{ONOO}^{-}$in live endothelial cells was studied. For this purpose, cells were first treated with $\mathrm{ONOO}^{-}$scavengers, ${ }^{36-38}$ and then were stimulated with SIN-1 or OGD. The results showed that neutralizing $\mathrm{ONOO}^{-}$with these scavengers significantly decreased SIN-1 or OGD induced B545b fluorescence (Fig. 4), suggesting the selectivity of B545b for $\mathrm{ONOO}^{-}$in live cells.

\section{In vivo real-time imaging of the $\mathrm{ONOO}^{-}$flux incurred by brain microvessel occlusion}

Having confirmed the sensitivity and selectivity of probe $\mathbf{B 5 4 5 b}$ for $\mathrm{ONOO}^{-}$in live cells, and its good BBB permeability, we then tested its ability to image $\mathrm{ONOO}^{-}$induced by brain vascular injury in mice. First, a photothrombosis-induced focal ischemia model was used to evaluate its ability to track the $\mathrm{ONOO}^{-}$flux incurred by brain microvessel occlusion in live mice. For this
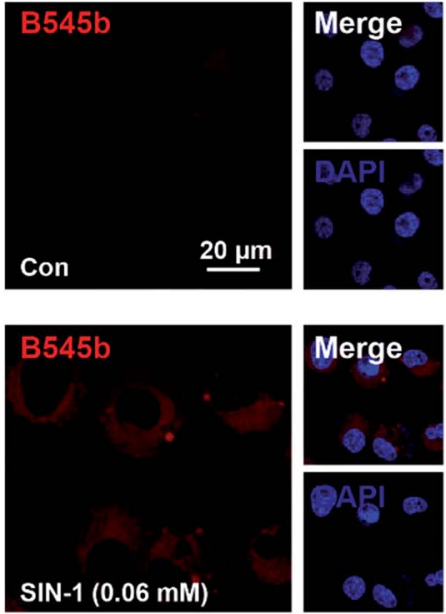

C
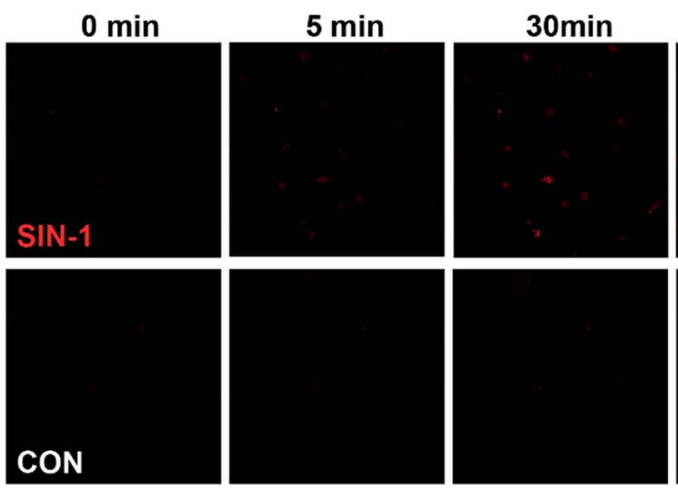

b
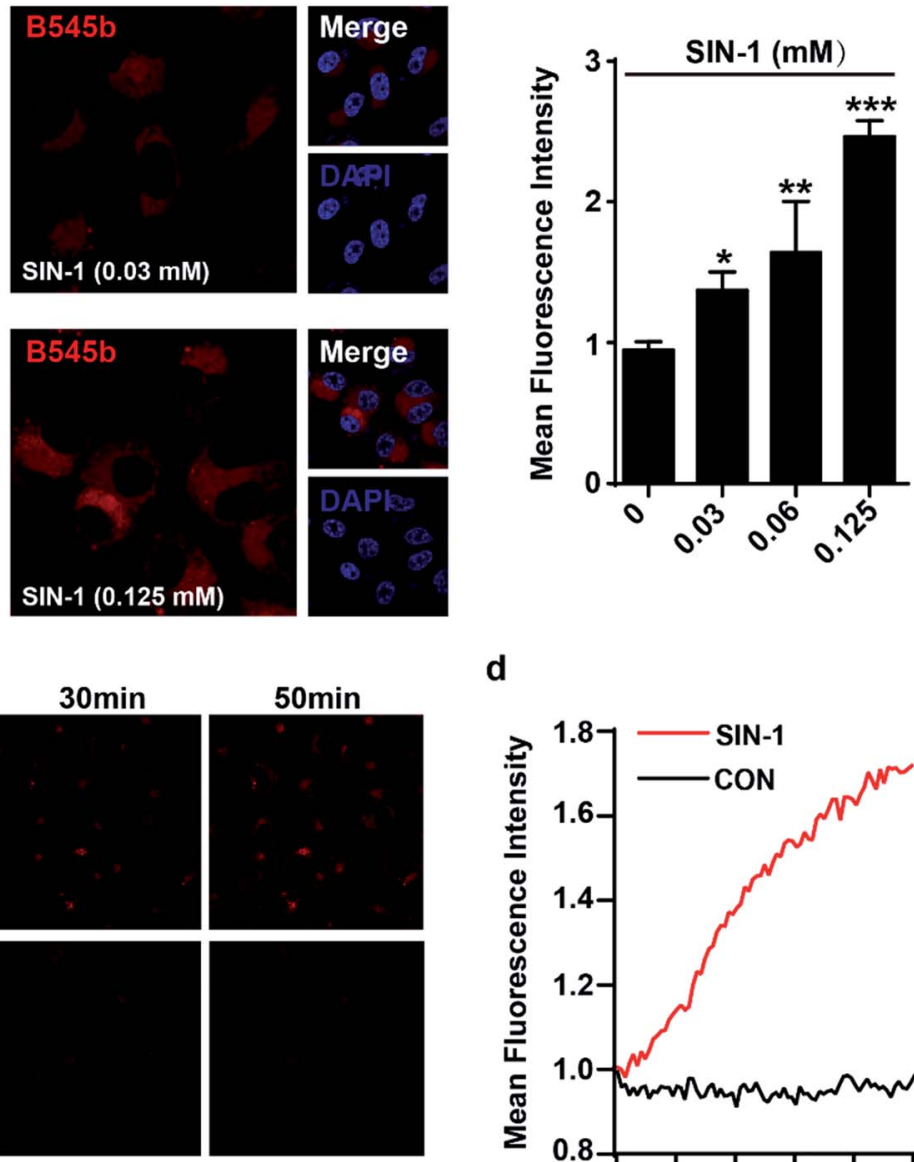

d

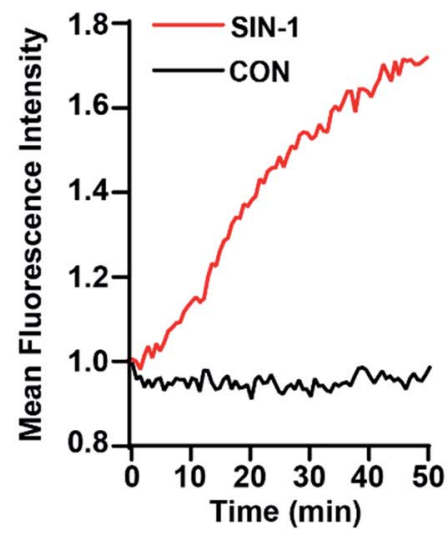

Fig. 3 Imaging SIN-1 induced ONOO- in EA.hy926 endothelial cells. (a) Cells were incubated with various doses of SIN-1 for $1 \mathrm{~h}$, and then stained with B545b $(0.5 \mu \mathrm{M}$ ) for 30 min. (b) Quantified B545b fluorescence in (a) (mean \pm S.E.M, $n=4$ ). (c) Time-lapse series of EA.hy926 cells first loaded with B545b and then stimulated with SIN-1 (1 mM). (d) Quantified B545b fluorescence in (c). Data are presented as a densitometric ratio change compared with control. DAPI $\left(4^{\prime}, 6\right.$-diamidino-2-phenylindole) counterstaining indicates nuclear localization (blue, $\lambda_{\mathrm{em}} 420-480, \lambda_{\mathrm{ex}}$ $405 \mathrm{~nm})$. B545b fluorescence was collected at 560-620 nm with $\lambda_{\text {ex }} 543 \mathrm{~nm}$. ${ }^{* * *} P<0.001, * * P<0.01$, and $* P<0.1$ versus control. 
a
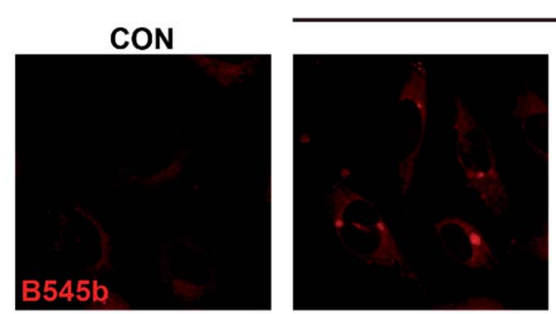

SIN-1 (1 mM)

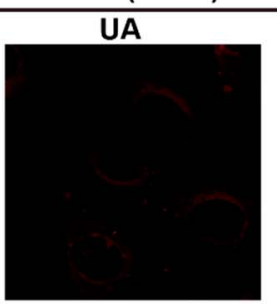

FeTPPS
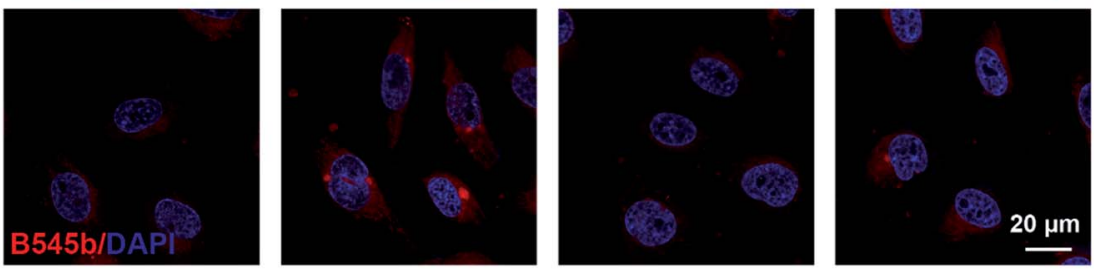

b
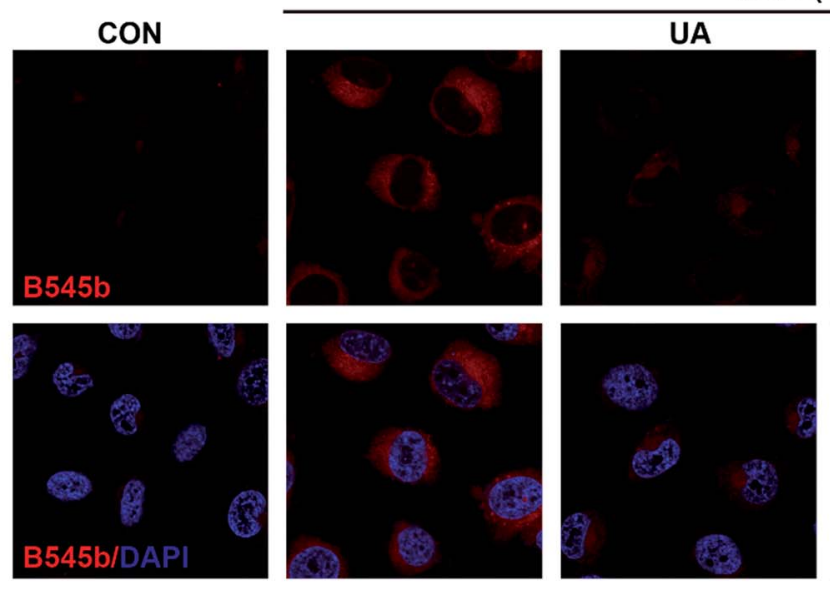

OGD (4 h)
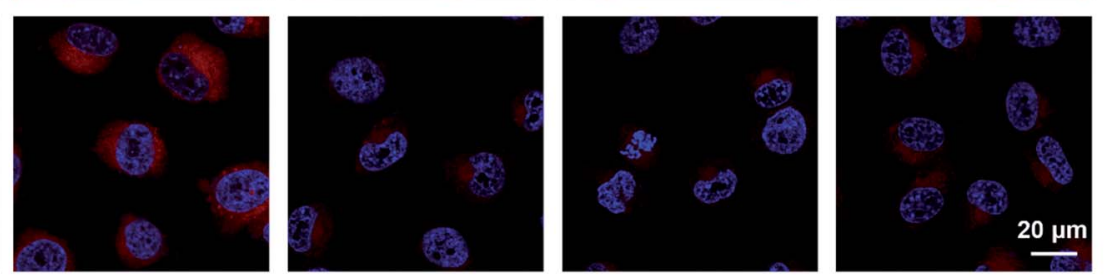

C

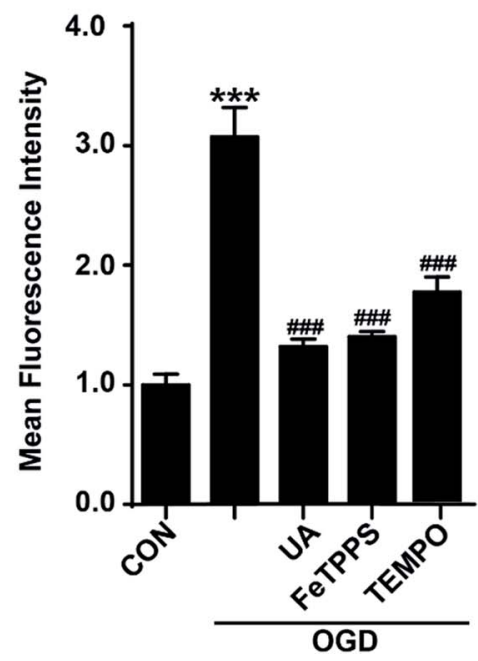

Fig. 4 Characterization of B545b specificity for $\mathrm{ONOO}^{-}$in endothelial cells. (a) Scavenging ONOO- with uric acid (100 $\left.\mu \mathrm{M}\right)$ or FeTPPS (1 $\mu \mathrm{M}$ ) inhibited SIN-1 induced B545b fluorescence. (b) Pretreating cells with uric acid (100 $\mu$ M). FeTPPS $(1 \mu M)$ or TEMPO (300 $\mu$ M) $1 \mathrm{~h}$ prior to OGD exposure ( 4 h) significantly suppressed B545b fluorescence. (c and d) Quantification of B545b mean fluorescence intensity (mean \pm S.E.M, $n=4$ ) in (a) and (b). Data are presented as a densitometric ratio change compared with control. DAPI counterstaining indicates nuclear localization (blue, $\lambda_{\mathrm{ex}} 405 \mathrm{~nm}$ and $\lambda_{\mathrm{em}} 420-480 \mathrm{~nm}$ ). B545b fluorescence was collected at 560-620 nm with $\lambda_{\mathrm{ex}} 543 \mathrm{~nm}$. $* * * P<0.001, * * P<0.01$ versus control, \#\#\#P<0.001, and \#\#P<0.01 versus SIN-1 or OGD. 
purpose, B545b was administered to mice through tail injection. One hour later, the mice were subjected to laser irradiation to induce microvessel occlusion and hence ischemic injury. ${ }^{39}$ Simultaneously B545b fluorescence in the occluded area was recorded using an in vivo two-photon laser scanning microscope (TPLSM) (Fig. 5a). As shown in Fig. 5b, which represents the time series of images from a continuous time-lapse movie (S1), B545b fluorescence was switched on simultaneously as the intravascular clot occurred, suggesting the immediate overproduction of $\mathrm{ONOO}^{-}$in response to clot formation. Interestingly, a trace amount of B545b fluorescence was also observed around the clotted region once the clot was formed but decreased soon as ischemia progressed, leaving the clotted site which is the major site of $\mathrm{ONOO}^{-}$overproduction. This result suggests that B545b can efficiently detect the burst of pathologic $\mathrm{ONOO}^{-}$and can visualize the dynamic changes of $\mathrm{ONOO}^{-}$in a temporally and spatially resolved way. The result also provides the direct proof for the instant overproduction of $\mathrm{ONOO}^{-}$in vascular injury.

\section{Detecting $\mathrm{ONOO}^{-}$production in the brain parenchyma after vascular injury}

Having confirmed the ability of B545b to track the burst of $\mathrm{ONOO}^{-}$in the clotted microvessel region, we then tested whether it could also detect $\mathrm{ONOO}^{-}$production in the brain parenchyma after vascular injury. For this purpose, an intraluminal middle cerebral artery occlusion (MCAO) model was used to induce cerebral infarction. ${ }^{40}$ Mice were administered with B545b through tail injection. $20 \mathrm{~min}$ later, the mice were subjected to transient MCAO of $0.5 \mathrm{~h}$ or $1 \mathrm{~h}$. An in vivo imaging system (IVIS) showed the MCAO-related activation of B545b fluorescence in the ipsilateral side of brain $0.5 \mathrm{~h}$ after MCAO (Fig. 6a). Ischemic injury evokes a cellular stress response, which involves the expression of FasL. As shown in Fig. 6b, an elevation in FasL expression was observed in MCAO mice, which indicated the damage induced by ischemic injury. ${ }^{\mathbf{4 1}}$ Observation of the tissues under a confocal microscope (Fig. 6c) revealed that $\mathbf{B 5 4 5 b}$ fluorescence increased mainly in the cortex and partly in the striatum of the left brain. There was no significant Nissl staining in the ipsilateral brain at least 0.5 and $1 \mathrm{~h}$ after ischemia (Fig. 6d). The control group with B545b administration but no MCAO treatment demonstrated negligible B545b fluorescence while B545b fluorescence was significantly improved in the brain tissues from MCAO-treated mice. This result suggests that a transient ischemia of $30 \mathrm{~min}$, even without reperfusion injury, is sufficient enough to cause nitrosative stress injury to the brain parenchyma. It should be noted
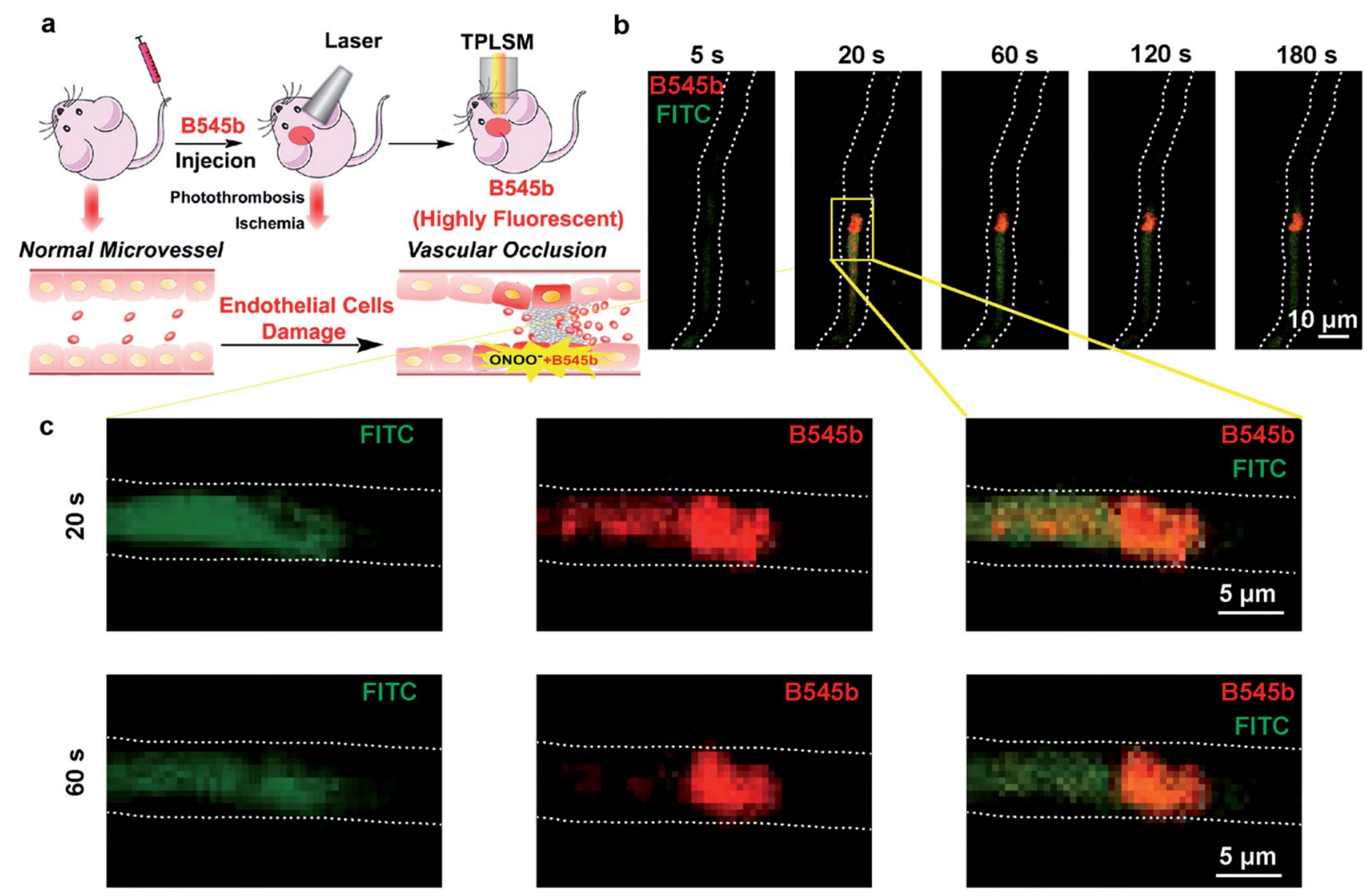

Fig. 5 In vivo real-time imaging of the endogenous $\mathrm{ONOO}^{-}$flux incurred by brain microvessel occlusion. (a) Schematic illustration of experimental methods for microvessel occlusion and imaging. First, probe B545b and FITC-dextran were intravenously injected into mice. Then, microvessel occlusion was induced by photothrombosis, and imaged using a two-photon laser scanning microscope (TPLSM). (b) The timeseries images representing individual frames from a continuous time lapse movie which showed the dynamic B545b fluorescence change in response to vascular occlusion in live mice. (c) Individual fluorescence of B545b and FITC in the photothrombosis model. 
a

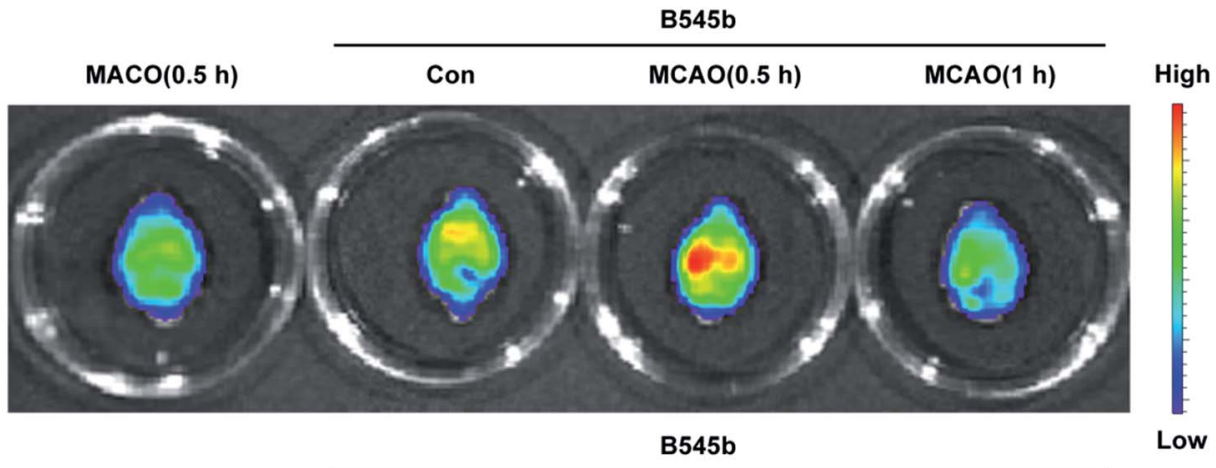

b
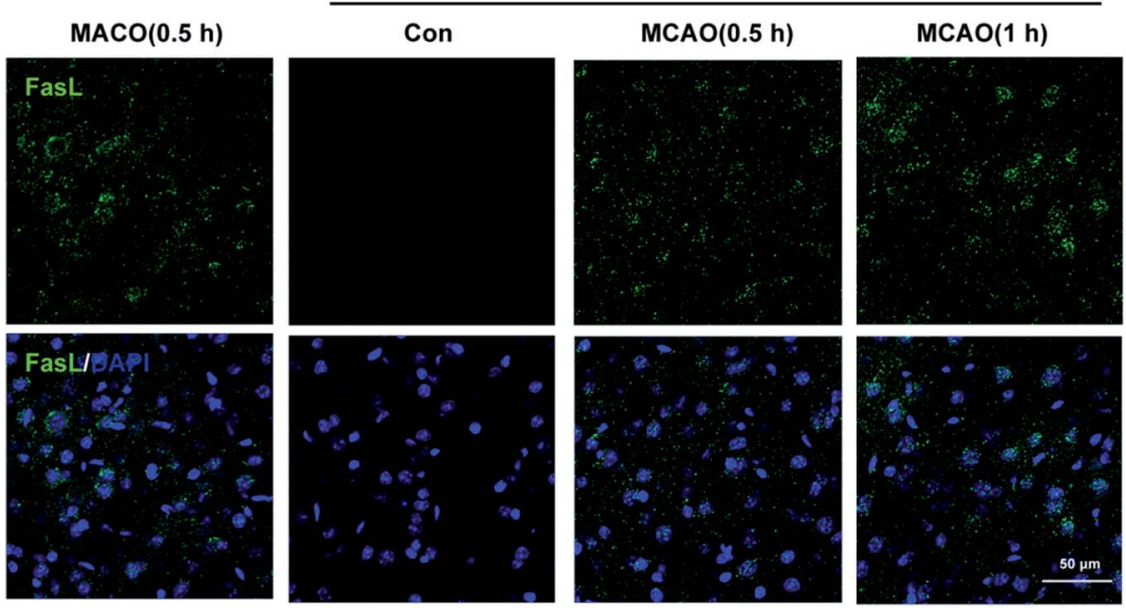

C
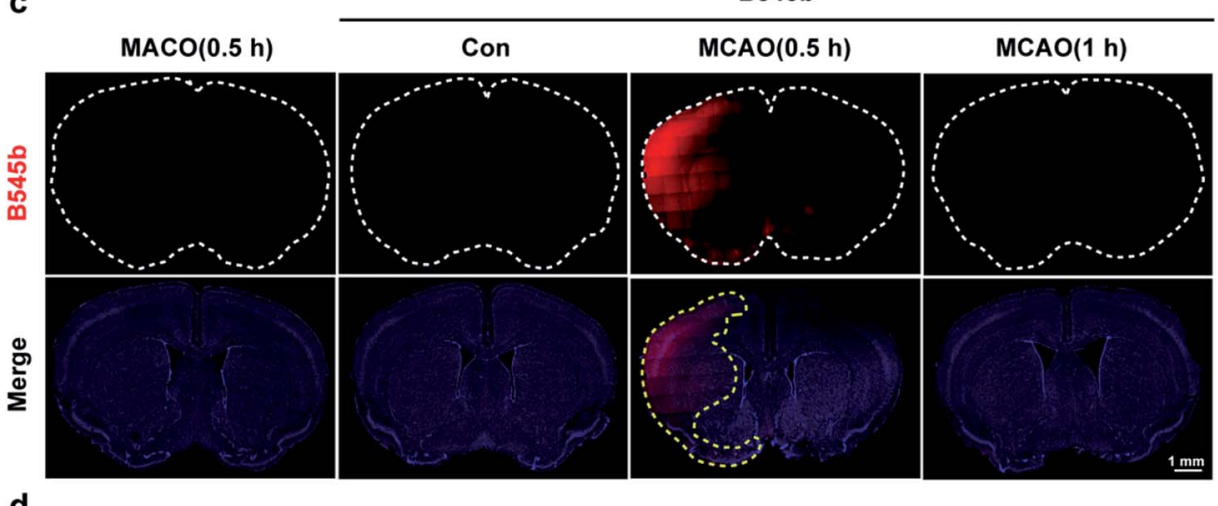

d

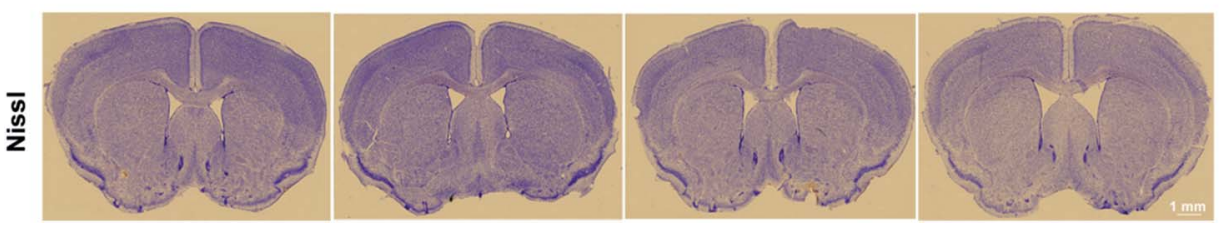

Fig. 6 Imaging of $\mathrm{ONOO}^{-}$accumulation after $\mathrm{MCAO}$ in the brain parenchyma. (a) Cerebral imaging and analysis. After B545b administration, the mice were treated with MCAO for 0.5 or $1 \mathrm{~h}$. The whole brains were scanned using a Maestro in vivo imaging system with a $480 \mathrm{~nm}$ excitation wavelength and a $560 \mathrm{~nm}$ filter. (b) Immunofluorescence staining of FasL (green) and DAPI (blue) in the brain after B545b injection and MCAO treatment. Scale bar $=50 \mu \mathrm{m}$. (c) Confocal imaging of brain slices with the B545b signal (red) and DAPI (blue). B545b fluorescence was collected at 500-540 nm with $\lambda_{\text {ex }}$ $488 \mathrm{~nm}$. B545b fluorescence significantly increased $0.5 \mathrm{~h}$ after MCAO in the ischemic brain. The white dotted lines show the brain sections and the yellow dotted lines show the ischemic region of brain. Scale bar $=1 \mathrm{~mm}$. (d) Nissl staining with the whole brain sections. Scale bar $=1 \mathrm{~mm}$.

that an MCAO of 60 min gave weaker B545b fluorescence than an MCAO of $30 \mathrm{~min}$, which may be due to the quick metabolism of B545b, as shown in Fig S12.†

\section{Conclusions}

In summary, using a physicochemical-property guided probe design strategy, we have developed a fluorogenic probe B545b 
for imaging $\mathrm{ONOO}^{-}$during the onset of stroke. The probe demonstrates an extremely weak background signal but significant brightness upon $\mathrm{ONOO}^{-}$stimulation, which makes it sensitive enough to trap endogenously produced pathological $\mathrm{ONOO}^{-}$. Moreover, due to its desirable physicochemical properties including its partition coefficient between water and oil and moderate water solubility, B545b can be administered intravenously to mice and it readily penetrates the brain blood barrier. These advantages make B545b sensitive enough to track the $\mathrm{ONOO}^{-}$flux in clotted microvessels, and to image ischemiainduced brain parenchymal damage. Facilitated by $\mathbf{B 5 4 5 b}$, immediate $\mathrm{ONOO}^{-}$overproduction accompanying thrombus formation was observed. Moreover, $\mathrm{ONOO}^{-}$overproduction in the brain parenchyma was also observed at the early stage of ischemia. This information should be helpful for the design of future anti-oxidant therapy for stroke. These results highlight B545b as a promising tool to probe the molecular role of $\mathrm{ONOO}^{-}$in the progression of neurovascular injury in stroke.

\section{Conflicts of interest}

There are no conflicts to declare.

\section{Ethical statement}

All animal procedures were performed in accordance with the Guidelines for Care and Use of Laboratory Animals of Zhejiang University and Experiments were approved by the Ethics Committees for Animal Experiments of Zhejiang University.

\section{Acknowledgements}

This work was supported in part by the National Natural Science Foundations of China (21778048, 21642007, 81473202, 81573411, and 81225022), the Zhejiang Provincial Natural Science Foundation of China (R18H300001 and Z16H310003), and the National Science \& Technology Major Project "Key New Drug Creation and Manufacturing Program" (2018ZX09711002010-004).

\section{Notes and references}

1 G. J. Hankey, Lancet, 2017, 389, 641-654.

2 A. Chamorro, U. Dirnagl, X. Urra and A. M. Planas, Lancet Neurol., 2016, 15, 869-881.

3 E. H. Lo, M. A. Moskowitz and T. P. Jacobs, Stroke, 2005, 36, 189-192.

4 N. Fukuyama, S. Takizawa, H. Ishida, K. Hoshiai, Y. Shinohara and H. Nakazawa, J. Cereb. Blood Flow Metab., 1998, 18, 123-129.

5 C. Szabo, H. Ischiropoulos and R. Radi, Nat. Rev. Drug Discovery, 2007, 6, 662-680.

6 A. Chamorro, J. Stroke, 2018, 20, 197-207.

7 S. Amaro, D. Canovas, M. Castellanos, J. Gallego, J. MartiFebregas, T. Segura and A. Chamorro, Int. J. Stroke, 2010, 5, 325-328.

8 R. Radi, Proc. Natl. Acad. Sci. U. S. A., 2018, 115, 5839-5848.
9 R. Radi, J. Biol. Chem., 2013, 288, 26464-26472.

10 J. Chan, S. C. Dodani and C. J. Chang, Nat. Chem., 2012, 4, 973-984.

11 D. Wu, A. C. Sedgwick, T. Gunnlaugsson, E. U. Akkaya, J. Yoon and T. D. James, Chem. Soc. Rev., 2017, 46, 71057123.

12 D. Cheng, Y. Pan, L. Wang, Z. Zeng, L. Yuan, X. Zhang and Y. T. Chang, J. Am. Chem. Soc., 2017, 139, 285-292.

13 J. B. Li, L. Chen, Q. Wang, H. W. Liu, X. X. Hu, L. Yuan and X. B. Zhang, Anal. Chem., 2018, 90, 4167-4173.

14 Y. Wu, A. Shi, Y. Li, H. Zeng, X. Chen, J. Wu and X. Fan, Analyst, 2018, 143, 5512-5519.

15 J. Zhang, X. Zhen, P. K. Upputuri, M. Pramanik, P. Chen and K. Pu, Adv. Mater., 2017, 29, 1604764.

16 C. Yin, H. Zhu, C. Xie, L. Zhang, P. Chen, Q. Fan, W. Huang and K. Pu, Adv. Funct. Mater., 2017, 27, 1700493.

17 J. Huang, J. Li, Y. Lyu, Q. Miao and K. Pu, Nat. Mater., 2019, 18, 1133-1143.

18 D. Cheng, J. Peng, Y. Lv, D. Su, D. Liu, M. Chen, L. Yuan and X. Zhang, J. Am. Chem. Soc., 2019, 141, 6352-6361.

19 A. J. Shuhendler, K. Pu, L. Cui, J. P. Uetrecht and J. Rao, Nat. Biotechnol., 2014, 32, 373-380.

20 T. Peng, X. Chen, L. Gao, T. Zhang, W. Wang, J. Shen and D. Yang, Chem. Sci., 2016, 7, 5407-5413.

21 Y. Li, X. Xie, X. Yang, M. Li, X. Jiao, Y. Sun, X. Wang and B. Tang, Chem. Sci., 2017, 8, 4006-4011.

22 T. Peng, N. K. Wong, X. Chen, Y. K. Chan, Z. Sun, J. J. Hu, J. Shen, H. El-Nezami and D. Yang, J. Am. Chem. Soc., 2014, 136, 11728-11734.

23 X. Xie, F. Tang, G. Liu, Y. Li, X. Su, X. Jiao, X. Wang and B. Tang, Anal. Chem., 2018, 90, 11629-11635.

24 S. A. Hitchcock and L. D. Pennington, J. Med. Chem., 2006, 49, 7559-7583.

25 K. Setsukinai, Y. Urano, K. Kakinuma, H. J. Majima and T. Nagano, J. Biol. Chem., 2003, 278, 3170-3175.

26 T. Peng, N. K. Wong, X. Chen, Y. K. Chan, Z. Sun, J. J. Hu, J. Shen, H. El-Nezami and D. Yang, J. Am. Chem. Soc., 2014, 136, 11728-11734.

27 X. Li, R. R. Tao, L. J. Hong, J. Cheng, Q. Jiang, Y. M. Lu, M. H. Liao, W. F. Ye, N. N. Lu, F. Han, Y. Z. Hu and Y. H. Hu, J. Am. Chem. Soc., 2015, 137, 12296-12303.

28 T. Peng, X. Chen, L. Gao, T. Zhang, W. Wang, J. Shen and D. Yang, Chem. Sci., 2016, 7, 5407-5413.

29 K. E. Knewtson, D. Rane and B. R. Peterson, ACS Chem. Biol., 2018, 13, 2595-2602.

30 D. Li, J. Cheng, C. K. Wang, H. Ying, Y. Hu, F. Han and X. Li, Chem. Commun., 2018, 54, 8170-8173.

31 T. Rohand, M. Baruah, W. Qin, N. Boens and W. Dehaen, Chem. Commun., 2006, 266-268.

32 A. M. Hakim and E. A. Shoubridge, Cerebrovasc. Brain Metab. Rev., 1989, 1, 115-132.

33 U. Bickel, NeuroRx, 2005, 2, 15-26.

34 J. H. Jang, O. I. Aruoma, L. S. Jen, H. Y. Chung and Y. J. Surh, Free Radical Biol. Med., 2004, 36, 288-299.

35 J. Xu, L. He, S. H. Ahmed, S. W. Chen, M. P. Goldberg, J. S. Beckman and C. Y. Hsu, Stroke, 2000, 31, 1744-1751. 
36 D. C. Hooper, S. Spitsin, R. B. Kean, J. M. Champion, G. M. Dickson, I. Chaudhry and H. Koprowski, Proc. Natl. Acad. Sci. U. S. A., 1998, 95, 675-680.

37 T. P. Misko, M. K. Highkin, A. W. Veenhuizen, P. T. Manning, M. K. Stern, M. G. Currie and D. Salvemini, J. Biol. Chem., 1998, 273, 15646-15653.

38 R. T. Carroll, P. Galatsis, S. Borosky, K. K. Kopec, V. Kumar, J. S. Althaus and E. D. Hall, Chem. Res. Toxicol., 2000, 13, 294-300.
39 M. Schroeter, S. Jander and G. Stoll, J. Neurosci. Methods, 2002, 117, 43-49.

40 N. Shioda, T. Ishigami, F. Han, S. Moriguchi, M. Shibuya, Y. Iwabuchi and K. Fukunaga, Neuroscience, 2007, 148, 221-229.

41 Y. M. Lu, R. R. Tao, J. Y. Huang, L. T. Li, M. H. Liao, X. M. Li, K. Fukunaga, Z. H. Hong and F. Han, J. Neuroinflammation, 2012, 9, 172. 